\title{
A Study on the Etiologic Agents of Bacterial Eye Infections in Khartoum State
}

\author{
Nuha M. Elhage ${ }^{1}$ Samia A .Gumaa ${ }^{2}$ \\ ${ }^{2}$ University of Medical Science and technology, Faculty of Medical laboratory, Microbiology department \\ ${ }^{2}$ Royal Care International Hospital, Microbiology Consultant (Khartoum)
}

\begin{abstract}
A total of 100 eye specimens were collected from patients with eye infection from different areas in Khartoum state of different age groups.The duration of study was from Junuary-Septemper 2008.

The bacterial isolates were related to the type of the eye infection, based on the diagnosis of the physicians which included conjunctivitis, keratitis, keratoconjuntivitis, cornial grafts infection, lacrimal gland infection and eye abscess. The gram-positive bacteria isolates were Staphylococcus aureus (34\%), Staphylococcus haemolyticus (12\%), Streptococcus pneumoniae(19\%) and Streptococcus viridians (8\%).

The gram-negative bacteria isolate were Haemophilus influenzae (15\%), Pseudomonas aeruginosa(7\%), Klebsilla pneumoniae(3\%), and Neisseria gonorrhea(2\%).The predominant bacteria isolated in this study was Staphylococcus spp.
\end{abstract}

Keywords: Bacterial isolates, eye infection, Antimicrobial susceptibility pattern, Sudan

\section{Introduction}

Conjunctivitis is an inflammation of the conjunctivae, which are the mucous membranes covering the white of the eyes and the inner side of the eyelids. It usually affects both eyes at the same time although it may start in one eye and spread to the other after a day or two. It may be asymmetrical, affecting one eye more than the other. There are many causes and the treatment will depend upon the cause. [1]

Bacterial conjunctivitis is a microbial infection involving the mucous membrane of the surface of the eye. This condition, which is usually a benign self-limited illness, sometimes can be serious or signify a severe underlying systemic disease. Occasionally, significant ocular and systemic morbidity may result [2].

Eye infections are eye ailments that are caused by bacterial, viral, or other microbial agent . The three most common causes of conjunctivitis are infection (infective conjunctivitis), allergic reactions and irritation (loose eyelash). Infective conjunctivitis is most commonly caused by bacteria and viruses. Viral conjunctivitis causes a watery discharge while the discharge from bacterial conjunctivitis contains pus[3]. People who wear contact lenses often find themselves getting some type of eye infection; this is due to the bacterial buildup from constantly wearing the lenses without proper disinfecting.

Some common eye infection are pink eye and Blepharitis which is an inflammation of the eyelid margins which can lead to discomfort and decreased the visual function[4].Trachoma is another eye infection ,more common in poor countries, that is spread easily and can lead to blindness[5] .

Eye infections usually require some type of medication, The indiscriminate use of antibiotics led to increase the risk of bacterial resistance to many commonly used antimicrobial medications. The emergence of bacterial resistance towards topical antimicrobial agents may also increase the risk of treatment failure with potentially serious consequences [6].

The aim of the study was to identify the causative agents bacterial pathogen common to ocular infection, and to assess the in vitro drug susceptibility patterns of these isolates in order to select the appropriates therapy for the patients. 


\section{Material and method}

\subsection{Study design}

This study is a cross-sectional hospital based study of patients suspected of bacterial conjunctivitis submitted to the Microbiology Laboratory Department.

\subsection{Study area}

The samples were taken from patient at the following hospitals in Khartoum state

- Ophthalmology Teaching Hospital.

- Yastabshiroon Medical Center.

- Makah Hospital

\subsection{Study population}

2.3.1 Selection criteria: Patients of different age groups who were diagnosed by the physician as having zacterial eye infection were included in this study.

\subsection{Sample size}

100 sample (48 Adult males, 45 adult females\&7children).

\subsection{Plane for Data Analysis and Processing}

Microsoft Excel SPSS (statistics package of social sciences).

\subsection{Ethical Consideration}

Informed consent was taken verbally from patient and their guardians in the study population and participation was entirely voluntary .Consent was ensured from the ethical consideration board committee of SUMASRI international review board.

\subsection{Sample collection}

Purulent material from the surface of the lower conjunctiva sac and inner can thus of the eye were collected by sterile swabs asepticsually and gently to avoid contamination from the skin. Two specimens collected from each patient, one for direct examination and other for culture. The samples were cultured immediately if possible and if not, put in (Aims transport media), transferred to the laboratory immediately for bacteriological examination.

\subsection{Direct examination}

All materials submitted for culture were smeared and examined directly by gram stain.

\subsection{Culture}

Because of the constant washing action of tears, the number of organism recovered from culture of certain eye may be relatively low, unless the clinical specimen is obviously purulent. Large inoculate were plated on the blood and chocolate agar plates incubated under increased carbon dioxide tension $(5-10 \% \mathrm{CO} 2)$. And on MacConkey agar, Mannitol Salt Agar . And incubated at $37^{\circ} \mathrm{C}$ for 48 hours. All plates were initially examined for growth after 24 hours and cultures with no growth were incubated for further 48 hours. After getting pure colonies, further identification were conducted by using standard microbiological techniques (conventail method), which include Gram stain, colony morphology and biochemical tests. Presumptive Gram negative bacteria were identified using triple sugar iron agar, citrate utilization test, lysine decarboxylase test, urease test and indole test and Gram positive bacteria were identified using catalase, coagulase, bacitracin and optchin sensitivity tests [7]. 


\subsection{Antimicrobial susceptibility test}

The antimicrobial susceptibility was performed on each identified bacterium using disc diffusion method on Muller Hinton agar based on national committee clinical and laboratory standard (NCCLS) guidelines.

\section{Result}

One hundred specimens collected, 48 from adult male and 45 from adult female while the other 7 were collected from children. Gram-positive organisms were isolated from 73 specimens and gram-negative organisms isolated from 27 specimens.

The most frequent isolated bacteria were Staphylococcus aureus(34\%), Staphylococcus haemolyticus(12\%), Streptococcus pneumoniae(19\%) and Streptococcus viridans(8\%), Haemophilus influenzae(15\%), Pseudomonas aeruginosa(7\%), Klebsilla pneumoniae(3\%), and Neisseria gonorrhea(2\%).

Three patients had mixed culture with two types of bacteria which were Haemophilus influenzae and Streptococcus pneumonia.

TABLE I: Shows Bacteria Isolated From Different Sites Of Eye Infection

\begin{tabular}{cccc}
\hline \hline Type of infection & Number of positive cases & Organism isolated & Number Organism isolated \\
\hline Conjunctivitis & 60 & Staphylococcus.aureus & 22 \\
& & Staphylococcus.haemolyticus & 7 \\
& & Streptococcus..viridans & 8 \\
& & Klebsella.pneumoniae & 3 \\
& & Streptococcus.pneumoniae & 11 \\
kertoconjunctivis & 5 & Haemophilus influenza & 4 \\
& & Pseudomonas aeruginosa & 2 \\
Lacrimal gland infection & Nesseria gonorrheae & 3 \\
& 34 & Pseudomonas aeruginosa & 11 \\
Corneal grafts & Staphylococcus.aurues & 5 \\
\hline \hline
\end{tabular}

Among the 73 of gram-positive isolates tested, non was sensitive to Penicillin ( $0 \%),(95 \%)$ were sensitive to Chloromphenicol and(100\%)were sensitive to Vancomycin respectively..

TABLE II: Shows Percentage Sensitivity Of Gram-Positive Isolates To Different Antimicrobial Agents.

\begin{tabular}{|c|c|c|c|c|c|}
\hline Antibiotics & $\begin{array}{c}\text { Staphylococcu } \\
\text { s } \\
\text { aureus }\end{array}$ & $\begin{array}{c}\text { Staphylococcus } \\
\text { haemolyticus }\end{array}$ & $\begin{array}{c}\text { Streptococcus } \\
\text { Viridans }\end{array}$ & $\begin{array}{l}\text { Streptococcus } \\
\text { pneumoniae }\end{array}$ & TOTAL \\
\hline Penicillin & $0 \%$ & $0 \%$ & $0 \%$ & $0 \%$ & $0 \%$ \\
\hline Tetracycline & 25 & $95 \%$ & $100 \%$ & $80 \%$ & $75 \%$ \\
\hline Fusidic acid & $80 \%$ & $50 \%$ & $30 \%$ & $25 \%$ & $46.25 \%$ \\
\hline Chloromphenicol & $80 \%$ & $100 \%$ & $100 \%$ & $100 \%$ & $95 \%$ \\
\hline Erythromycin & $14 \%$ & $6 \%$ & $0 \%$ & $55 \%$ & $18.75 \%$ \\
\hline Gentamicin & $25 \%$ & $80 \%$ & $100 \%$ & $0 \%$ & $51.25 \%$ \\
\hline Septrin & $25 \%$ & $0 \%$ & $75 \%$ & $0 \%$ & $25 \%$ \\
\hline Vancomycin & $100 \%$ & $100 \%$ & $100 \%$ & $100 \%$ & $100 \%$ \\
\hline
\end{tabular}


The 27 gram-negative isolates, were sensitive to Amikacin, Chlormphenicol, Ceftriaxone and Gentamicin $(93.7 \%),(92.5 \%),(88.75 \%)$ and $(87.5 \%)$ respectively.

TABLE III: Shows Percentage Sensitivity Of Gram-Negative Isolates To Different Antimicrobial Agents.

\begin{tabular}{lccccc}
\hline \hline Antibiotics & Psudomonas.aeruginosa & Klebsilla.pneumoniae & Haemophilus.influnzae & Neisseria.gonorrhea & Total \\
\hline Ampicillin & $0 \%$ & $100 \%$ & $100 \%$ & $0 \%$ & $50 \%$ \\
Chloromphinicol & $70 \%$ & $100 \%$ & $100 \%$ & $100 \%$ & $92.5 \%$ \\
Fusidic acid & $0 \%$ & $0 \%$ & $75 \%$ & $0 \%$ & $18.75 \%$ \\
Ceftriaxone & $80 \%$ & $100 \%$ & $75 \%$ & $100 \%$ & $88.75 \%$ \\
Gentamycin & $75 \%$ & $100 \%$ & $75 \%$ & $100 \%$ & $87.5 \%$ \\
Tetracycline & $100 \%$ & $0 \%$ & $0 \%$ & $0 \%$ & $25 \%$ \\
Amikacin & $100 \%$ & $100 \%$ & $75 \%$ & $100 \%$ & $93.75 \%$ \\
Ciprofloxacin & $100 \%$ & $100 \%$ & $90 \%$ & $0 \%$ & $72.5 \%$ \\
\hline \hline
\end{tabular}

\section{Discussion}

The eye and its associated structure are uniquely predisposed to infection by various microorganisms, mainly bacteria, viruses, fungi and rarely parasite (Forbes et al.2007)[8] .

This study was conducted to isolate and identify bacteria associated with eye infection in human, the study was also conducted to determine the antimicrobial sensitivity of the isolated bacteria.

Eye swabs were taken from patients diagnosed by physicians as having bacterial eye infection.

In this study many organism were isolated from patient with conjunctivitis. This include Staphylococcus aureus, Staphylococcus haemolyticus, Streptococcus pneumoniae, Streptococcus viridans Haemophilus influenza ,Neisseria gonorrhea, Pseudomonas aeruginosa and Klebsiella pneumonia

In the study done by (Shiferaw et al) ${ }^{[9]}$ in 2015,160 specimens were taken from patients with external ocular infection, $95(59.4 \%)$ were positive for different bacterial species. The predominant isolates were belong to gram positive bacteria $93.7 \%$ (89/95), which is supported by the present study .Staphylococcus aureus was isolated from $(23.6 \%, n=21 / 89)$, which is much less than the isolated rate of the same organism in the present study which was(34\%).

Also Streptococcus pneumoniae was isolated from $(11.2 \%, n=10 / 89)$ where as in this study were $(19 \%)$.the rate of isolation of Klebsella pneumonia was similar to our present study which were (2.9\%) and (3\%) .we agree that conjunctivitis was the dominant type of eye infection (43.1\%) in their study, and (60\%)in present study.

In this study, the association of Pseudomonas aeruginosa with conjunctivitis was in agreement with a similar study carried out in patients with conjunctivitis in Bangalore (2014) by (Hemavathi, et al) ${ }^{[10]}$ with the same percentage (7\%). The isolation of Streptococcus pneumoniae and Homophiles influenzae in mixed infection in the study by Forbes et al.(2007)[8] was similar to our finding.

The drug susceptibility patterns of Gram positive cocci bacterial isolates showed a $100 \%$ sensitivity pattern to vancomycin, which was similar to (Shirferaw et al) ${ }^{[9]}$ However, all of the isolates were resistant to penicillin, similar to another study which showed $90-100 \%$ resistance(Hemavathi ${ }^{[10]}$, Tesfaye ${ }^{[11]}$.

Most of Gram negative isolates were sensitive to Amikacin and chloromphenicol (93.75\%) (92.5\%) respectively, but resist to tetracycline and fusidic acid. Both Gram positive cocci and Gram negative bacilli were highly susceptible to chloromphenicol.

\section{Conclusion}

In case of bacterial eye infection it is very important to provide accurate diagnosis, prompt treatment and management to prevent a bad prognosis that may lead to serious complication.

It can be concluded that:

- The Gram-positive bacteria especially Staphylococcus species were the most common causative agents of bacterial eye infection in Sudan. 
- Vancomycin is drug of choice for Gram-positive isolates . All of the isolated were sensitive to it.

- Amikacin and Chloromphenicol showed favorable in vitro activity against the majority of gram negative pathogens.

- Successful treatments can only be achieved through early treatment after antibiotic sensitivity.

- Resistance rates indicate the need for continuous surveillance and for motoring studies.

\section{References}

[1] Haraze Evert ,Paul S.Little,Peter WF .Smith "Arnado-Mised control of management stratiges for acute infection conjunctivitis in general practice"BMJ (2006): 10.1136.

[2] Gopinathan U, Sharma S, Garg P, Rao G. Review of epidemiological features, microbiological diagnosis and treatment outcome of microbial keratitis: Experience of over a decade. Indian J Ophthalmol. 2009;57(4):273-9. https://doi.org/10.4103/0301-4738.53051

[3] Marilyn H and Charles S. Conjunctivitis: Bacterial, Viral, Allergic and Other Types. All about vision.com. Access Media group LLC, March 2014.

[4] Azari AA, Barney NP. Conjunctivitis A Systematic Review of Diagnosis and Treatment. JAMA. 2013;310:1721-9. https://doi.org/10.1001/jama.2013.280318

[5] Meneghim RL1, Viveiros MM1, Galindo-Ferreiro A2, Padovani CR3, Schellini SA1,2. Sequelae from Epidemic Viral Conjunctivitis Can Be Associated with Inflammatory Trachoma in Schoolchildren? Semin Ophthalmol. 2016 Sep $14: 1-4$.

[6] Sharma S. Antibiotics and Resistance in Ocular Infections. Indian J Med Microbiol. 2011;29:218-22. https://doi.org/10.4103/0255-0857.83903

[7] Cheesbrough M. District Laboratory Practice in Tropical Countries Part I. 2nd ed. New York: Cambrige University Press; 2006. https://doi.org/10.1017/CBO9780511543470

[8] Forbes, B. A.; Sahm, D. F. and Weissfeld, A S, Bailley and Scott's. Diagnostic Microbiology. 12th ed., Mosby, London, U. k. (2007)

[9] Birtukan Shiferaw, Baye Gelaw, Abate Assefa, Yared Assefa and Zelalem Addis. Bacterial isolates and their antimicrobial susceptibility pattern among patients with external ocular infections at Borumeda hospital, Northeast Ethiopia .BMC Ophthalmology201515:103 https://doi.org/10.1186/s12886-015-0078-z DOI: $10.1186 / \mathrm{s} 12886-015-0078-\mathrm{z}$. https://doi.org/10.1186/s12886-015-0078-z

[10] Hemavathi,1 Pooja Sarmah,2 and Poornima Shenoy3. Profile of Microbial Isolates in Ophthalmic Infections and Antibiotic Susceptibility of the Bacterial Isolates: A Study in an Eye Care Hospital, Bangalore. Journal of Clinic Diagnostic Research. 2014 January; 8(1): 23-25.

[11] Tesfaye T, Beyene G, Gelaw Y, Bekele S, Saravanan M. Bacterial Profile and Antimicrobial Susceptibility Pattern of External Ocular Infections in Jimma University Specialized Hospital, Southwest Ethiopia. Am J Ophthalmology. 2013;1:13-20 https://doi.org/10.12691/ajidm-1-1-3 